\title{
Arginase 1 Regulation of Nitric Oxide Production Is Key to Survival of Trophic Factor-Deprived Motor Neurons
}

\author{
Alvaro G. Estévez, ${ }^{1,2}$ Mary Anne Sahawneh, ${ }^{1,2}$ Philipp S. Lange, ${ }^{1,2}$ Narae Bae, ${ }^{1,2}$ Mariela Egea, ${ }^{1,2}$ and Rajiv R. Ratan ${ }^{1,2}$ \\ ${ }^{1}$ Burke Medical Research Institute, White Plains, New York 10605, and ${ }^{2}$ Department of Neurology and Neurosciences, Weill Medical School of Cornell \\ University, New York, New York 10021
}

\begin{abstract}
When deprived of trophic factors, the majority of cultured motor neurons undergo nitric oxide-dependent apoptosis. However, for reasons that have remained unclear, $30-50 \%$ of the motor neurons survive for several days without trophic factors. Here we hypothesize that the resistance of this motor neuron subpopulation to trophic factor deprivation can be attributed to diminished nitric oxide production resulting from the activity of the arginine-degrading enzyme arginase. When incubated with nor-N $\mathrm{N}^{\mathrm{G}}$-hydroxy-nor-L-arginine (NOHA), the normally resistant trophic factor-deprived motor neurons showed a drop in survival rates, whereas trophic factor-treated neurons did not. NOHA-induced motor neuron death was inhibited by blocking nitric oxide synthesis and the scavenging of superoxide and peroxynitrite, suggesting that peroxynitrite mediates NOHA toxicity. When we transfected arginase 1 into motor neurons to see whether it alone could abrogate trophic factor deprivation-induced death, we found that its forced expression did indeed do so. The protection afforded by arginase 1 expression is reversed when cells are incubated with NOHA or with low concentrations of nitric oxide. These results reveal that arginase acts as a central regulator of trophic factor-deprived motor neuron survival by suppressing nitric oxide production and the consequent peroxynitrite toxicity. They also suggest that the resistance of motor neuron subpopulations to trophic factor deprivation may result from increased arginase activity.
\end{abstract}

Key words: nitric oxide; motor neurons; arginase; peroxynitrite; apoptosis; trophic factor deprivation

\section{Introduction}

Motor neuron survival in vitro and in vivo is known to depend on the continuous supply of trophic factors (Oppenheim, 1996, 1997; Sendtner et al., 2000; Wiese et al., 2004). A variety of trophic factors can prevent development-associated motor neuron death, maintain survival of adult motor neurons after injury when delivered in vivo, and prevent motor neuron death in vitro (Oppenheim, 1996, 1997; Sendtner et al., 2000; Wiese et al., 2004). Motor neuron death after ventral root avulsion and induced by deprivation of trophic factors in culture involves the induction of nitric oxide synthase (NOS) and production of the potent oxidant peroxynitrite, which in turn triggers apoptosis (Estévez et al., 1998a, 2000; Martin et al., 1999, 2005). New evidence suggests that a balance of trophic and death-signaling pathways regulates motor neuron survival (Raoul et al., 1999, 2000, 2002). For example, tumor necrosis factor and/or the Fas pathway appear to regulate motor neuron death resulting from ventral root avulsion (Ugolini et al., 2003; Martin et al., 2005). Fas activation also plays a role in motor neuron apoptosis induced by trophic factor deprivation in culture and induces motor neuron

Received Feb. 17, 2006; revised June 15, 2006; accepted June 24, 2006.

This work was supported by National Institutes of Health/National Institute of Neurological Disorders and Stroke Grants NS36761 and NS42834 (A.G.E.) and NS40591 and NS4623 (R.R.R.). We thank Dr. Christopher E. Henderson (Columbia University, New York, NY) for his suggestions during the preparation of this manuscript. We also thank Drs. Kimberly McGhee and Wayne Kleinman for their assistance in the preparation of this manuscript.

Correspondence should be addressed to Dr. Alvaro G. Estévez, Burke Research Institute, 785 Mamaroneck Avenue, White Plains, NY 10605. E-mail: age2002@med.cornell.edu.

DOI:10.1523/JNEUROSCI.0728-06.2006

Copyright $\odot 2006$ Society for Neuroscience $\quad$ 0270-6474/06/268512-05\$15.00/0 death in the presence of trophic factors (Raoul et al., 1999, 2002). Motor neuron death induced by either trophic factor deprivation or death factors shares a common final pathway, one that involves transcriptional induction of neuronal NOS, nitric oxide production, and peroxynitrite formation. Accordingly, under conditions of trophic factor deprivation or Fas activation stimulation, motor neuron survival can be effectively sustained by inhibiting nitric oxide production and scavenging superoxide (Estévez et al., 1998a, 2000; Cassina et al., 2002; Raoul et al., 2002). However, for reasons that have eluded investigation, 30$50 \%$ of trophic factor-deprived motor neurons survive for $3 \mathrm{~d}$ in culture (Henderson et al., 1994; Pennica et al., 1996; Estévez et al., 1998a, 2000; Raoul et al., 1999, 2002).

Nitric oxide is produced from arginine by the three NOS isoforms. Nitric oxide production is regulated by calcium and posttranslational modification as well as at the level of enzyme transcription (Stuehr, 1999). Because arginine is the only substrate capable of donating the guanidine group necessary for nitric oxide production, modulating the levels of arginine can also regulate nitric oxide production. (Wiesinger, 2001; Herrera and Garvin, 2005). Arginase 1, an L-arginine-degrading enzyme, can influence the production of nitric oxide by limiting the pool of L-arginine available for NOS (Mori and Gotoh, 2000; Wiesinger, 2001). Although all motor neurons deprived of trophic factor express neuronal NOS, the levels of nitrotyrosine immunoreactivity are highly variable (Estévez et al., 1998a; Raoul et al., 2002). We hypothesized that, in the surviving motor neuron subpopulations, nitric oxide produc- 
tion could be impaired by low levels of arginine caused by the high basal activity of arginase.

\section{Materials and Methods}

Cell culture. Motor neuron cultures were prepared as described previously (Henderson et al., 1995; Raoul et al., 1999; Estévez et al., 2000). Briefly, rat embryo [embryonic day 15 (E15)] spinal cords were dissected and the dorsal half removed. Ventral cords were chopped into pieces and incubated in modified F10 medium (without calcium and magnesium, and with sodium pantothenate) supplemented with $0.05 \%$ trypsin for 15 min at $37^{\circ} \mathrm{C}$, followed by mechanical dissociation. Motor neurons were then purified by centrifugation on an OptiPrep (Axis Shield PoC, Oslo, Norway) cushion followed by a step of immunoaffinity separation using IgG 192 against p75 neurotrophin receptor (Chandler et al., 1984). Motor neurons were plated at a density of 280 cells $/ \mathrm{cm}^{2}$ in four-well plates precoated with polyornithine and laminin in neurobasal medium supplemented with B27 supplement, glutamate, glutamine, 3-mercaptoethanol, and antibiotics, as described previously (Pennica et al., 1996; Estévez et al., 2000). Cultures were maintained at $37^{\circ} \mathrm{C}$ in a $5 \%$ $\mathrm{CO}_{2}$ humidified atmosphere. More than $95 \%$ of the cells were immunoreactive for the motor neuron markers Islet 1/2 (4D5 monoclonal antibody from the Developmental Studies Hybridoma Bank, Iowa City, IA) (Ericson et al., 1992; Tsuchida et al., 1994) and p75 neurotrophin receptor, as described previously (Estévez et al., 1998a).

Transfection. Electroporation of motor neurons was performed as described previously (Raoul et al., 2002), with minor modifications. Briefly, motor neurons were purified from E15 rat embryos by density cushion centrifugation followed by immunoaffinity separation. Cells were collected and rinsed on a BSA cushion. Motor neurons were resuspended at a density of 50,000 cells in $100 \mu \mathrm{l}$ of electroporation buffer (in mM): 20 HEPES, $125 \mathrm{NaCl}, 5 \mathrm{KCl}, 1.5 \mathrm{MgCl}_{2}$, and 10 glucose, $\mathrm{pH}$ 7.4. The pEGFP.C1 (Clontech, Mountain View, CA) and the pEIF/Myc-His C (Invitrogen, Carlsbad, CA) vectors containing the gene for the arginase 1 (5 $\mu \mathrm{g}$ each) were added to the cells in the electroporation buffer and incubated for 15-20 min. The cells were transferred to a $1 \mathrm{~mm}$ gap cuvette (BTX, San Diego, CA) and transfected (three pulses of $5 \mathrm{~ms}$ at $200 \mathrm{~V}$ at 1-s intervals) using an Electro Square Porator ECM830 (BTX). After electroporation, the cells were diluted in Neurobasal medium supplemented with B27, glutamate, glutamine, 2-mercaptoehtanol, horse serum, and a combination of trophic factors. The cells were plated in a four-well plate precoated with poly-ornithine/laminin and incubated overnight before removal of the trophic factors. Motor neuron survival was determined $3 \mathrm{~d}$ after removal of the trophic factors by counting all green fluorescent protein (GFP)-positive neurons with neurites longer than 4 soma in diameter in two diagonals for each well. All neurons that showed fluorescence also had neurites. The number of surviving neurons in cultures transformed and treated with a combination of brain-derived neurotrophic factor (BDNF) (1 ng/ml), glial-derived neurotrophic factor (GDNF) $(0.1 \mathrm{ng} / \mathrm{ml})$, and cardiotrophin $1(10 \mathrm{ng} / \mathrm{ml})$ was used at $100 \%$ as an interexperimental standardization.

Immunoflurescence. Cultures were fixed with paraformaldehyde plus glutaraldehyde (Sigma, St. Louis, MO) on ice for $15 \mathrm{~min}$ or incubated with methanol at $20^{\circ} \mathrm{C}$ for $5 \mathrm{~min}$ before the staining was performed, as described previously (Estévez et al., 1998a; Lee et al., 2003).

Cell viability. Motor neuron survival was assessed by counting all phase-bright cells displaying intact neurites longer than four cells in diameter under $10 \times$ magnification on the surface of two-well diameters (in four-well plates). All values are expressed as a percentage of the number of motor neurons present in parallel cultures maintained with BDNF $(1 \mathrm{ng} / \mathrm{ml})$ or with a combination of trophic factors [BDNF $(1 \mathrm{ng} / \mathrm{ml})$, GDNF (0.1 ng/ml) (both from R\&D Systems, Minneapolis, MN), and cardiotrophin 1 (10 ng/ml; Alomone Labs, Jerusalem, Israel)] (Estévez et al., 1998a,b, 2000).

Nitric oxide measurements. Motor neurons were purified and plated on 96-well plates (Greiner Bio-One, Kremsmuenster, Austria) precoated with poly-ornithine/laminin in Neurobasal medium supplemented with B27, glutamate, glutamine, 2-mecaptoethanol and horse serum at the density of 1500 motor neuron per well. Motor neurons were cultured with or without trophic factors for $3 \mathrm{~d}$ before performing the measure-

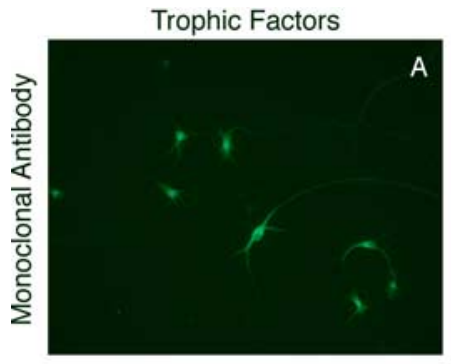

Trophic Factor Deprivation
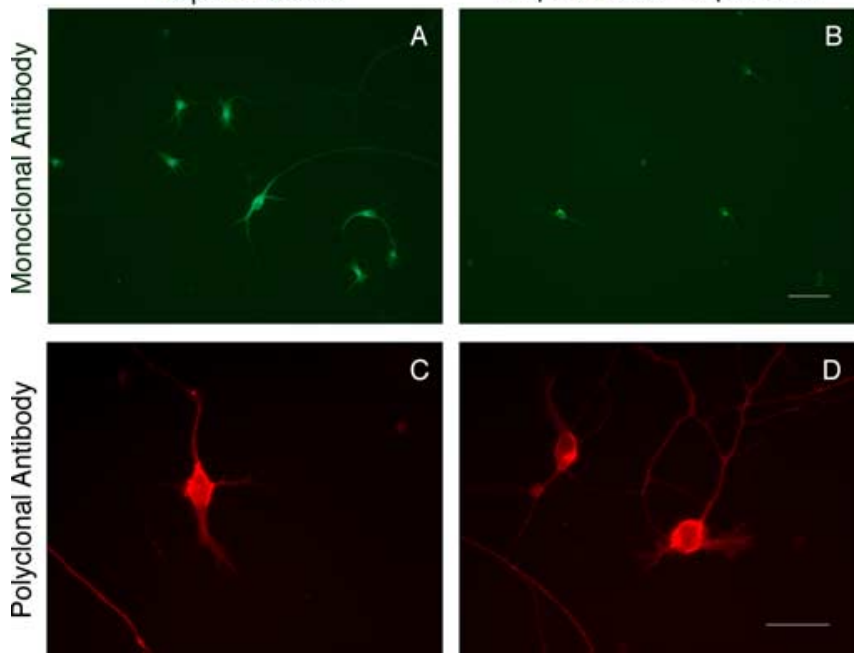

Figure 1. Motor neurons were cultured for $3 \mathrm{~d}$ with $(\boldsymbol{A}, \boldsymbol{C})$ and without $(\boldsymbol{B}, \boldsymbol{D})$ trophic factors and stained for arginase 1 using mouse monoclonal $(\boldsymbol{A}, \boldsymbol{B})$ and rabbit polyclonal $(\boldsymbol{C}, \boldsymbol{D})$ antibodies as described in Materials and Methods. The images were captured after $3 \mathrm{~d}$ in culture. Scale bars: (in $\boldsymbol{B}) \boldsymbol{A}, \boldsymbol{B}, 100 \mu \mathrm{m}$; (in $\boldsymbol{D}) \boldsymbol{C}, \boldsymbol{D}, 50 \mu \mathrm{m}$.

ments for nitric oxide. Nitric oxide was measured by 4 -amino-5methylamino-2', $7^{\prime}$-difluorofluorescein (DAF-FM) diacetate (Invitrogen, Eugene, OR) fluorescence. The cultures were preincubated with the corresponding treatments for $1 \mathrm{~h}$ before removing the culture media and replace it with $200 \mu \mathrm{l}$ of $\mathrm{L} 15$ without phenol red containing $10 \mu \mathrm{M}$ DAF-FM diacetate and the corresponding treatments. The cultures were incubated for 1 additional hour at $37^{\circ} \mathrm{C}$. At that time, hemoglobin at a final concentration of $7.5 \mathrm{mg} / \mathrm{ml}$ was added to quench nonspecific florescence. The plates were read using a Flash Cytometer high-throughput imaging system (Trophos, Marseilles, France). The digitalized images were analyzed, and the mean florescence intensity per cell in each well was obtained using the Tina software (Trophos).

Statistical analysis. Reported values are the mean \pm SD for the number of samples indicated for each experiment. Values were analyzed by oneway ANOVA and the Kruskal-Wallis nonparametric test followed by Dunn's multiple-comparison test, unless indicated otherwise. All statistics and graphs were performed using Prism 4.0c (GraphPad Software, San Diego, CA). $p<0.05$ was considered significant.

\section{Results}

To determine whether resistant motor neuron subpopulations express arginase 1, we used polyclonal (Lee et al., 2003) and monoclonal (BD Biosciences Pharmingen, San Jose, CA) antibodies to arginase 1, which render the same results. After $3 \mathrm{~d}$ in culture, all trophic factor-treated motor neurons and the surviving motor neurons cultured without trophic factors were immunoreactive for arginase 1 with immunoreactivity concentrated in the motor neuron soma and proximal neurites (Fig. 1).

Rat embryo motor neurons constitutively express the endothelial isoform of the nitric oxide synthase (Estévez et al., 1998b). The role of arginase 1 in the regulation of nitric oxide production by motor neurons was studied in 3-d-old cultures. Inhibition of arginase activity by arginase nor- $\mathrm{N}^{\mathrm{G}}$-hydroxy-nor-L-arginine (NOHA) in trophic factor-treated cultures induced an $\sim 75 \%$ increase in nitric oxide production as determined by DAF-FM fluorescence (Fig. 2). In the absence of trophic factors, there was no detectable production of nitric oxide by DAF-FM, as was expected because of the diffusion limited reaction of nitric oxide with superoxide to form peroxynitrite (Estévez et al., 1998a, 2000).

The $3 \mathrm{~d}$ of survival in culture of trophic factor-deprived motor 


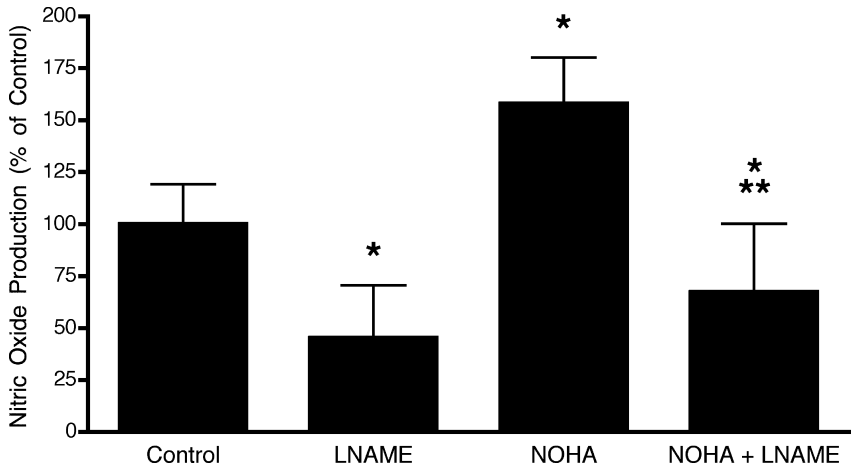

Figure 2. Production of nitric oxide by motor neurons in culture. After $3 \mathrm{~d}$ in culture, the culture medium was replaced, and the cells were incubated in $\mathrm{L} 15$ without phenol red supplemented with DAF-FM (Control) or treated with $100 \mu \mathrm{m}$ nitro-L-arginine (LNAME), $1 \mu \mathrm{M} \mathrm{NOHA,}$ or $1 \mu \mathrm{m}$ NOHA plus $100 \mu \mathrm{m}$ LNAME for $2 \mathrm{~h}$ before the fluorescence was measured as described in Materials and Methods. The $100 \%$ is the fluorescence intensity in the cultures without treatment. The values are the mean $\pm S D$ of three experiments performed in quintuplicate. ${ }^{*} p<$ 0.001 versus Control; ${ }^{* *} p<0.001$ versus NOHA (one-way ANOVA followed by Bonferroni's post test).

neurons was half that of the motor neurons cultured in the presence of BDNF (1 ng/ml) (Fig. 3A). To investigate the role of arginase 1 in the survival of motor neurons cultured with and without trophic factors, we inhibited the enzyme activity with NOHA (Bachem Bioscience, King of Prussia, PA). Arginase 1 inhibition had no effect on the survival of motor neurons cultured with BDNF. In contrast, inhibition of arginase 1 with NOHA decreased the survival of trophic factor-deprived motor neurons in a dose-dependent manner, with an $\mathrm{EC}_{50}$ of $0.7 \mu \mathrm{M}$ $(0.4-1 \mu \mathrm{M}, 95 \%$ confidence interval) and the maximum effect achieved at $\sim 70 \mu \mathrm{M}$ (Fig. $3 A$ ).

Inhibition of arginase 1 leads to an increase in the intracellular concentration of arginine, thereby providing a substrate for increased nitric oxide production. Because motor neuron death induced by trophic factor deprivation is dependent on the simultaneous production of nitric oxide and superoxide, we tested the effects of NOS inhibitors and scavengers of superoxide and peroxynitrite on NOHA toxicity. The toxic effects of NOHA were suppressed by the nitric oxide synthase inhibitor nitro-L-arginine methyl ester. That protective effect, however, was reversed when DETANONOate $[(Z)-1-[N$-(2-aminoethyl)- $N$-(2-ammonioethyl)amino] diazen-1-ium-1,2-diolate] $(20 \mu \mathrm{M})$ was added to the culture medium to generate low steady-state concentrations of NO (nitric oxide) $(\sim 100 \mathrm{nM})$ (Fig. $3 B)$. The superoxide and peroxynitrite scavengers FeTCPP (iron porphyrin) and MnTBAP [Mn(III) tetra(4-benzoic acid) porphyrin chloride] also reversed the toxic effects of NOHA, thereby increasing motor neuron survival.

To further test the role of arginase 1 in the regulation of motor neuron survival, motor neurons were transfected with a vector expressing GFP or with two vectors, one expressing GFP and the other arginase 1. Electroporation of motor neurons with the vector expressing GFP resulted in a transfection efficacy of $\sim 70 \%$ of the surviving neurons. Trophic factor deprivation-induced motor neuron death was prevented by the overexpression of arginase 1 (Fig. 4) but not by the overexpression of GFP. The protection afforded by arginase 1 overexpression was reversed by NOHA and nitric oxide (Fig. 4). Overexpression of arginase 1 had no effect on the survival of BDNF-treated motor neurons (Fig. 4). Together, these results demonstrate that arginase inhibitioninduced motor neuron death, like growth factor deprivationinduced death, is mediated via a peroxynitrite pathway.

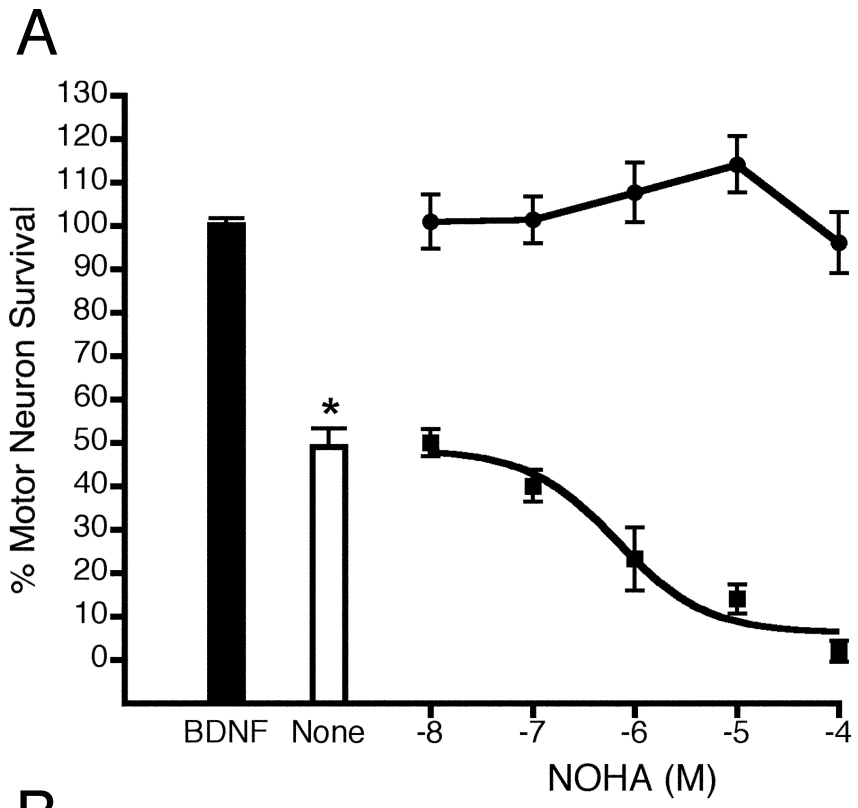

$\mathrm{B}$

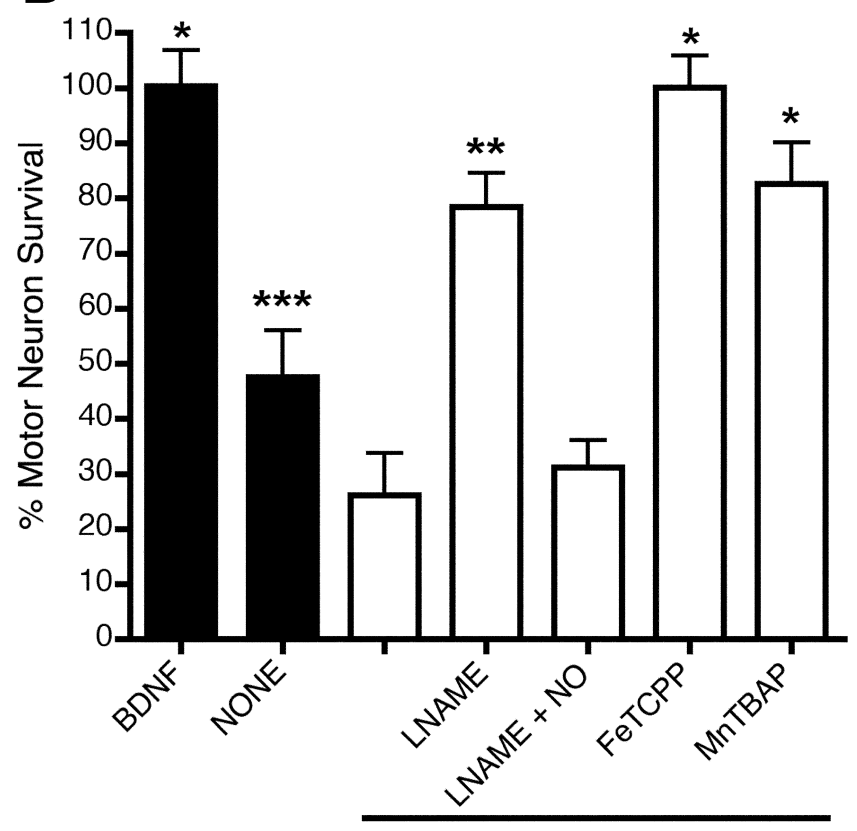

NOHA

Figure 3. $A, \mathrm{NOHA-induced} \mathrm{death} \mathrm{of} \mathrm{trophic} \mathrm{factor-deprived} \mathrm{motor} \mathrm{neurons.} \mathrm{Motor} \mathrm{neurons}$ were cultured for $3 \mathrm{~d}$ with BDNF (filled bar) and without trophic support (open bar). NOHA was added during plating alone at the indicated concentrations $(\square)$ or in combination with $1 \mathrm{ng} / \mathrm{ml}$ $\operatorname{BDNF}(-)$. The relation between NOHA concentration and motor neuron survival was fitted to a line with slope of 0 for BDNF and to a sigmoid curve using nonlinear regression (goodness of fit $R^{2}=0.91$ with 57 degrees of freedom). NOHA reduced motor neuron survival in a dosedependent manner with an $\mathrm{EC}_{50}$ of $0.7 \pm 0.7 \mu \mathrm{m}$ (mean $\pm \mathrm{SD}$ ), the top best-fit value equal to $48 \pm 10 \%$ (mean $\pm S D$ ), and the bottom best-fit value equal to $6 \pm 10 \%$. Values are the mean $\pm S D$ of three independent experiments performed in quadruplicate. ${ }^{*} p<0.001$ versus BDNF analyzed first by ANOVA and then by the Newman-Keuls multiple-comparison test. $\boldsymbol{B}$, Inhibition of NOS and scavenging of superoxide and peroxynitrite reversed the toxic effects of NOHA. Motor neurons were cultured for $3 \mathrm{~d}$ with (open bars) or without (filled bar) NOHA (1 $\mu \mathrm{m})$. Trophic factor-deprived motor neurons were incubated with NOHA (1 $\mu \mathrm{m})$ or with NOHA and either nitro-L-arginine methyl ester (LNAME) (100 $\mu \mathrm{M})$, iron porphyrin (FeTCPP) $(10 \mu \mathrm{M})$, or Mn(III) tetra(4-benzoic acid) porphyrin chloride (MnTBAP) $(100 \mu \mathrm{M})$. Motor neuron survival was determined after $3 \mathrm{~d}$ in culture. Values are the mean $\pm S D$ of three independent experiments performed in quadruplicate. ${ }^{*} p<0.001$ versus NOHA; ${ }^{* *} p<0.01$ versus NOHA; ${ }^{* * *} p<$ 0.05 versus $\mathrm{NOHA}$. 


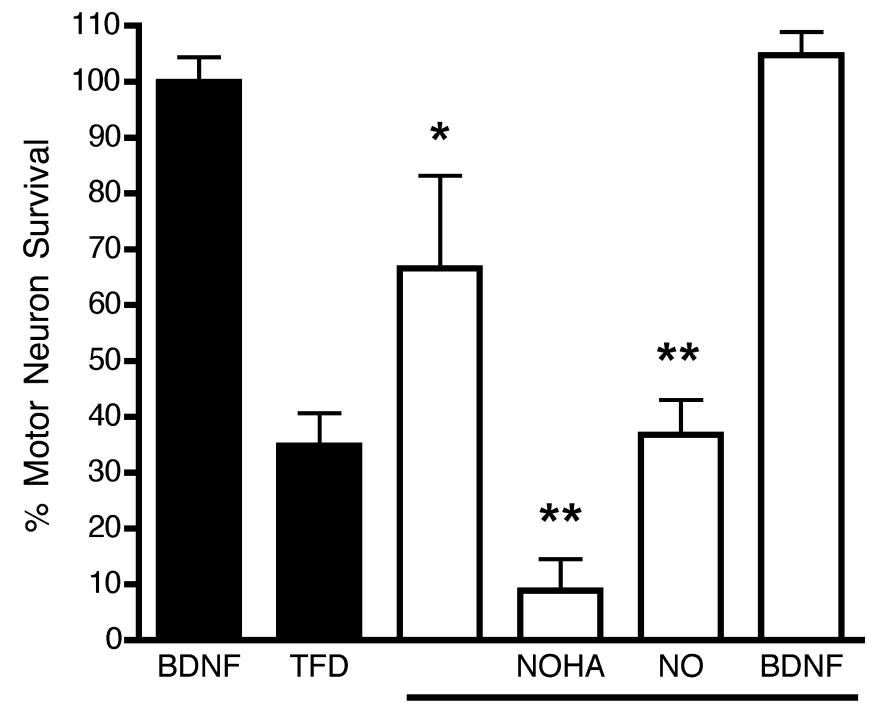

Arginase 1

Figure 4. Overexpression of arginase prevented motor neuron death induced by trophic factor deprivation. Motor neurons were electroporated with the PEGFP.C1 vector alone (filled bars) or in combination with the pEIF/Myc-His C vector containing the gene for the arginase 1 (open bars). Motor neurons were incubated in the presence of trophic factors overnight. Motor neuron survival was determined $3 \mathrm{~d}$ after trophic factor deprivation by counting all fluorescent cells. The protection provided by overexpression of arginase 1 was reversed by incubation with $1 \mu \mathrm{M}$ NOHA or $20 \mu \mathrm{M}$ DETANONOate [(Z)-1-[N-(2-aminoethyl)- $N$-(2-ammonioethyl)amino]diazen-1-ium-1,2-diolate]. Overexpression of arginase 1 did not affect the survival of motor neurons incubated with $1 \mathrm{ng} / \mathrm{ml}$ BDNF at the moment of changing the medium. Values are the mean \pm SD from at least three independent experiments performed in quadruplicate. ${ }^{*} p<0.01$ versus trophic factor deprivation (TFD); ${ }^{* *} p<0.01$ versus arginase 1.

\section{Discussion}

A central unanswered question in motor neuron biology has been why $30-50 \%$ of neurons in culture show resistance to trophic factor deprivation-induced or death receptor-induced death (Henderson et al., 1993, 1994; Milligan et al., 1994, 1995; Pennica et al., 1996; Estévez et al., 1998a, 2000; Raoul et al., 1999). When deprived of trophic support or after stimulation of death receptors, motor neurons die by apoptosis, which is dependent on de novo synthesis of neuronal NOS, nitric oxide production, and peroxynitrite formation (Estévez et al., 1998a, 2000; Martin et al., $1999,2003,2005)$. Herein we propose a model to explain the differential vulnerability of motor neurons after trophic factor deprivation. Because arginase 1 can regulate nitric oxide formation by modulating levels of arginine (Fig. 2), its only endogenous precursor, the ability of nitric oxide to achieve the threshold level required for toxicity will depend on the balance between neuronal NOS and arginase activity in the cell (Mori and Gotoh, 2000; Wiesinger, 2001; Lange et al., 2004). This model would predict that inhibition of arginase 1 in trophic factor-deprived motor neurons blocks the resistance, leaving motor neurons vulnerable to die (Fig. $3 A$ ), and that it would do so via the production of nitric oxide and peroxynitrite (Fig. $3 B$ ). The model also predicts that forced expression of arginase 1 would prevent trophic factorinduced death, a resistance that could in turn be overcome by the exogenous addition of nitric oxide (Fig. 4). Although we have not examined whether forced expression of arginase 1 in motor neurons affects neuronal NOS activity by limiting the availability of arginine or by expressing the enzyme (Lee et al., 2003), all of our results confirm that the inhibition of nitric oxide by arginase 1 accounts for the resistance seen in surviving populations of motor neurons deprived of trophic factors.
At least two pathways have been proposed to explain the protection afforded by arginase. Polyamines, small molecules with regularly spaced cation charges, are known to influence a diversity of biological activities including cell proliferation, regeneration, and death (Lange et al., 2004; Seiler and Raul, 2005; Takano et al., 2005). Arginase can increase polyamine synthesis by catalyzing the hydrolysis of arginine to ornithine and urea. Ornithine is then converted to putrescine by ornithine decarboxylase. In a previous study, we demonstrated that transduction of DRG neurons with a viral vector encoding arginase 1 rendered these neurons resistant to the neurite outgrowth-suppressing effects of myelin, presumably by enhancing polyamine synthesis and putrescine production (Cai et al., 2002). Another putative pathway of neuroprotection by arginase results from the intracellular depletion of arginine (Lee et al., 2003; Lange et al., 2004). Critically low levels of arginine can activate the serine/threonine kinase GCN2 to phosphorylate the translational initiation factor $2 \alpha$ (eif $\alpha$ ), which has been associated with cell survival (Sattlegger et al., 2004; Holcik and Sonenberg, 2005). However, it is unlikely that either of these pathways is the dominant mechanism of arginase protection against trophic factor deprivation in cultured motor neurons, because the addition of exogenous nitric oxide nearly completely reverses the salutary effects of arginase, and inhibition of arginase leads to a nitric oxide-peroxynitritedependent death.

Based on these findings, it can be predicted that the motor neuron subpopulation showing resistance to trophic factor deprivation-induced apoptosis will be those showing the highest arginase activity and that this resistance will be achieved via the inhibition of nitric oxide and peroxynitrite formation. In summary, the results presented here reveal a key role for arginase in the regulation of nitric oxide metabolism and of cultured motor neuron survival, warranting additional in vitro and in vivo studies on the role of arginase in motor neuron survival.

\section{References}

Cai D, Deng K, Mellado W, Lee J, Ratan RR, Filbin MT (2002) Arginase I and polyamines act downstream from cyclic AMP in overcoming inhibition of axonal growth MAG and myelin in vitro. Neuron 35:711-719.

Cassina P, Peluffo H, Pehar M, Martinez-Palma L, Ressia A, Beckman JS, Estévez AG, Barbeito L (2002) Peroxynitrite triggers a phenotypic transformation in spinal cord astrocytes that induces motor neuron apoptosis. J Neurosci Res 67:21-29.

Chandler CE, Parsons LM, Hosang M, Shooter EM (1984) A monoclonal antibody modulates the interaction of nerve growth factor with PC12 cells. J Biol Chem 259:6882-6889.

Ericson J, Thor S, Edlund T, Jessell TM, Yamada T (1992) Early stages of motor neuron differentiation revealed by expression of homeobox gene Islet-1. Science 256:1555-1560.

Estévez AG, Spear N, Manuel SM, Radi R, Henderson CE, Barbeito L, Beckman JS (1998a) Nitric oxide and superoxide contribute to motor neuron apoptosis induced by trophic factor deprivation. J Neurosci 18:923-931.

Estévez AG, Spear N, Thompson JA, Cornwell TL, Radi R, Barbeito L, Beckman JS (1998b) Nitric oxide-dependent production of cGMP supports the survival of rat embryonic motor neurons cultured with brain-derived neurotrophic factor. J Neurosci 18:3708-3714.

Estévez AG, Sampson JB, Zhuang Y-X, Spear N, Richardson GJ, Crow JP, Tarpey MM, Barbeito L, Beckman JS (2000) Liposome-delivered superoxide dismutase prevents nitric oxide-dependent motor neuron death induced by trophic factor withdrawal. Free Radic Biol Med 28:437-446.

Henderson CE, Camu W, Mettling C, Gouin A, Poulsen K, Karihaloo M, Rullamas J, Evans T, McMahon SB, Armanini MP, Berkemeier L, Phillips HS, Rosenthal A (1993) Neurotrophins promote motor neuron survival and are present in embryonic limb bud. Nature 363:266-270.

Henderson CE, Phillips HS, Pollock RA, Davies AM, Lemeulle C, Armanini M, Simpson LC, Moffet B, Vandlen RA, Koliatsos VE, Rosenthal A 
(1994) GDNF: a potent survival factor for motoneurons present in peripheral nerve and muscle. Science 266:1062-1064.

Henderson CE, Bloch-Gallego E, Camu W (1995) Purified embryonic motoneurons. In: Neural cell culture: a practical approach (Cohen J, Wilkin G, eds), pp 69-81. Oxford: IRL.

Herrera M, Garvin JL (2005) Recent advances in the regulation of nitric oxide in the kidney. Hypertension 45:1062-1067.

Holcik M, Sonenberg N (2005) Translational control in stress and apoptosis. Nat Rev Mol Cell Biol 6:318-327.

Lange PS, Langley B, Lu P, Ratan RR (2004) Novel roles for arginase in cell survival, regeneration, and translation in the central nervous system. J Nutr 134:2812S-2817.

Lee J, Ryu H, Ferrante RJ, Morris Jr SM, Ratan RR (2003) Translational control of inducible nitric oxide synthase expression by arginine can explain the arginine paradox. Proc Natl Acad Sci USA 100:4843-4848.

Martin LJ, Kaiser A, Price AC (1999) Motor neuron degeneration after sciatic nerve avulsion in adult rat evolves with oxidative stress and is apoptosis. J Neurobiol 40:185-201.

Martin LJ, Price AC, McClendon KB, Al-Abdulla NA, Subramaniam JR, Wong PC, Liu Z (2003) Early events of target deprivation/axotomyinduced neuronal apoptosis in vivo: oxidative stress, DNA damage, p53 phosphorylation and subcellular redistribution of death proteins. J Neurochem 85:234-247.

Martin LJ, Chen K, Liu Z (2005) Adult motor neuron apoptosis is mediated by nitric oxide and Fas death receptor linked by DNA damage and p53 activation. J Neurosci 25:6449-6459.

Milligan CE, Oppenheim RW, Schwartz LM (1994) Motoneurons deprived of trophic support in vitro require new gene expression to undergo programmed cell death. J Neurobiol 25:1005-1016.

Milligan CE, Prevette D, Yaginuma H, Homma S, Cardwell C, Fritz LC, Tomaselli KJ, Oppenheim RW, Schwartz LM (1995) Peptide inhibitors of the ICE protease family arrest programmed cell death of motoneurons in vivo and in vitro. Neuron 15:385-393.

Mori M, Gotoh T (2000) Regulation of nitric oxide production by arginine metabolic enzymes. Biochem Biophys Res Commun 275:715-719.

Oppenheim RW (1996) Neurotrophic survival molecules for motoneurons: an embarrassment of riches. Neuron 17:195-197.

Oppenheim RW (1997) Related mechanisms of action of growth factors and antioxidants in apoptosis: an overview. Adv Neurol 72:69-78.

Pennica D, Arce V, Swanson TA, Vejsada R, Pollock RA, Armanini M, Dudley
K, Phillips HS, Rosenthal A, Kato AC, Henderson CE (1996) Cardiotrophin-1, a cytokine present in embryonic muscle, supports longterm survival of spinal motoneurons. Neuron 17:63-74.

Raoul C, Henderson CE, Pettmann B (1999) Programmed cell death of embryonic motoneurons triggered through the Fas death receptor. J Cell Biol 147:1049-1062.

Raoul C, Peetmann B, Henderson CE (2000) Active killing of neurons during development and following stress: a role for p $75^{\text {NTR }}$ and Fas? Curr Opin Neurobiol 10:111-117.

Raoul C, Estévez AG, Nishimune H, Cleveland DW, deLapeyrière O, Henderson CE, Haase G, Pettmann B (2002) Motoneuron death triggered by a specific pathway downstream of Fas: potentiation by ALS-linked SOD1 mutations. Neuron 35:1067-1083.

Sattlegger EE, Swanson MJMJ, Ashcraft EAEA, Jennings JLJL, Fekete RARA, Link AJAJ, Hinnebusch AGAG (2004) YIH1 is an actin-binding protein that inhibits protein kinase GCN2 and impairs general amino acid control when overexpressed. J Biol Chem 279:29952-29962.

Seiler NN, Raul FF (2005) Polyamines and apoptosis. J Cell Mol Med 9:623-642.

Sendtner M, Pei G, Beck M, Schweizer U, Wiese S (2000) Developmental motoneuron cell death and neurotrophic factors. Cell Tissue Res 301:71-84.

Stuehr DJ (1999) Mammalian nitric oxide synthases. Biochimica Biophys Acta 1411:217-230.

Takano KK, Ogura MM, Nakamura YY, Yoneda YY (2005) Neuronal and glial responses to polyamines in the ischemic brain. Curr Neurovasc Res 2:213-223.

Tsuchida T, Ensini M, Morton SB, Baldassare M, Edlund T, Jessell TM, Pfaff SL (1994) Topographic organization of embryonic motor neurons defined by expression of LIM homeobox genes. Cell 79:957-970.

Ugolini G, Raoul C, Ferri A, Haenggeli C, Yamamoto Y, Salaun D, Henderson CE, Kato AC, Pettmann B, Hueber AO (2003) Fas/tumor necrosis factor receptor death signaling is required for axotomy-induced death of motoneurons in vivo. J Neurosci 23:8526-8531.

Wiese S, Beck M, Karch C, Sendtner M (2004) Signalling mechanisms for survival of lesioned motoneurons. Acta Neurochir (Wien) [Suppl] $89: 21-35$.

Wiesinger H (2001) Arginine metabolism and the synthesis of nitric oxide in the nervous system. Prog Neurobiol 64:365. 